\title{
Remote Data Monitoring for CDF
}

H.A. Kippenhan, Jr., W. Lidinsky, G. Roediger, M. Leininger, J. Patrick, E. Wicklund and G.P. Yeh

Fermi National Accelerator Laboratory

P.O. Box 500, Batavia, Illinois 60510

FERMILAB-Conf-95/359

\author{
F. Abe, Y. Morita and Y. Watase \\ KEK, National Laboratory for High Energy Physics \\ Tsukuba, Ibaraki 305, Japan
}

T. Watts

Rutgers University, Physics Department

Box 849, Piscataway, New Jersey 08855

S. Aota, S. Kim and M. Shimojima

University of Tsukuba

Tsukuba, Ibaraki 305, Japan

P.S. Chang

Institute of Physics, Academia Sinica

Taipei, Taiwan 11529

November 1995

Proceedings of Computing in High Energy Physics 1995, (CHEP '95), Rio de Janeiro, Brazil, September 18-22, 1995. 


\section{Disclaimer}

This report was prepared as an account of work sponsored by an agency of the United States Government. Neither the United States Government nor any agency thereof, nor any of their employees, makes any warranty, express or implied, or assumes any legal liability or responsibility for the accuracy, completeness, or usefulness of any information, apparatus, product, or process disclosed, or represents that its use would not infringe privately owned rights. Reference herein to any specific commercial product, process, or service by trade name, trademark, manufacturer, or otherwise, does not necessarily constitute or imply its endorsement, recommendation, or favoring by the United States Government or any agency thereof. The views and opinions of authors expressed herein do not necessarily state or reflect those of the United States Government or any agency thereof. 


\title{
REMOTE DATA MONITORING FOR CDF
}

\author{
H.A. Kippenhan Jr., W. Lidinsky, G. Roediger
}

M. Leininger. J. Patrick, E. Wicklund. G.P. Yeh

Fermi National Accelerator Laboratory, Batavia. IL. 60510

F. Abe, Y. Morita, Y. Watase

IIEK. National Laboratory for High Energy Physics. Tsukuba. Ibaraki 305, Japan

T. Watts

Rutgers University, Physics Dept, Box 849. Piscataway. NJ 08855

S. Aota. S. Kim, M. Shimojima

Lniversity of Tsukuba. Tsukuba. Ibaraki 305, Japan

P.S. Chang, G.P. Yeh

Institute of Physics, Academia Sinica, Taipei, Taiwan 11529

\begin{abstract}
Remote data monitoring from the physicists' home institutions has become an important issue in large international experiments to ensure high performance of the detectors and high quality of data and scientific results. The CDF experiment is a collaboration of $\mathbf{4 5 0}$ physicists from 36 institutions in the U.S., Japan, Canada, Italy and Taiwan. Future experiments at Fermilab, CERN and elsewhere will be even larger, and will be performed over a period of order 10 years. The ability of collaborators at remote sites to monitor the increasingly complex detectors and feed the results back into the data acquisition process will be of great importance We report on the status and performance of remote monitoring from Japan of the CDF experiment in Batavia Illinois. We also discuss feasibilities for modest Remote Control Rooms.
\end{abstract}

\section{Introduction}

Remote data monitoring from the physicists' home institutions has become an important issue in large international experiments to ensure high performance of the detectors and high quality of data and scientific results. The CDF experiment is a collaboration of 450 physicists from 36 institutions in the US, Japan, Canada, Italy and Taiwan. Future experiments at Fermilab, CERN and elsewhere will be even larger, and will be performed over a period of order 10 years. The ability of collaborators at remote sites to monitor the increasingly complex detectors and feed the results back into the data acquisition process will be of great importance.

Information about the collaboration and experiment can be found on the Web at: http://www-cdf.fnal.gov/ 
CDF data acquisition monitoring includes accelerator status, detector statistics. collision rates, trigger, event pictures. and selecting events to perform online and offline analyses. High-end networking enables data access and monitoring at remote sites. Packet video conferencing provides communication between collaborators at the remote site and collaborators in the CDF control room (on-site) at Fermilab. or at other remote institutions.

\section{Data monitoring in the CDF Control room}

Each CDF shift crew has consisted of 5 shift persons: SciCo(Scientific Coordinator). $\mathrm{RD}$ (Research Division) Operator, ACE, and $2 \mathrm{CO}$ 's(Consumer Operators). The job of the SciCo is coordination and management of the shift in physical and technical aspects. As this job needs detailed realtime information about the status of accelerator and data taking, it is better done by local staff at Fermilab. It should be improved in the future using video conferencing and other communication systems.

The job of the ACE is operation of the DAQ system. Normally the ACE is operating Run Control. The task can be done remotely, but he or she sometimes needs to reset some devices or swap modules to recover from troubles. Such operations need remote control mechanisms or the help of local engineers or shift crew.

The CO's jobs are operation of consumer processes, monitoring the status of detector system, safety walkthrough, etc. Some of this work can be done remotely, but other tasks must be done on site at Fermilab. However the work can be divided because there are 2 CO's in a shift. So the current scheme is to study performing a part of the CO's job from Japan.

\section{Remote data monitoring test}

Today, CDF data monitoring is done from remote sites via high speed data communications and a video link with the CDF Control Room. This operation is dubbed "Remote Shift" when a non-Fermi site takes over control room functions.

- 1) Monitoring the CDF detector and data acquisition, including: detector statistics, collision rates, trigger, event pictures, and diagnostic physics results.

- 2) Monitoring Fermilab accelerator status.

-3) Working with collaborators in the control room and remote institutions.

- 4) Selecting event data and performing near-on-line and off-line data analysis.

A short report of the Remote Shift taken at Tsukuba, Japan can be found at: http://hepsg l.px.tsukuba.ac.jp/topics/remoteshift/main.html.

\subsection{Equipment in CDF Control Room at Fermilab used to support remote moni- toring}

CDF DAQ system, Control Room VAX workstations, 1 SGI Indy workstation with 64 Mbytes of memory, 1 Panasonic video camera, a microphone and two small Apple speakers. 


\subsection{Equipment used at Tsukuba}

1 SGI Indy workstation(FW8C-1G32) with a camera (CANON VC-C1), a video visualizer (CANON RE-650) and a microphone (AUDIO-TECHINICA AT8601 ), 3 NCD X-terminals, I VAX 4000/90 workstation. I DEC Alpha workstation 2100/A500MP. 1 Laser Printer (QMS1060).

\subsection{Equipment used at $\mathrm{KEK}$}

VAX Station 4000/90 (19" display), HP 9000 712/80 (19" display), Fujitsu Sun 4/2 (19" display), Hitachi NCD X-Terminal (21" display), Mac II (12" display).

Before starting consumer processes, turning off security check of $\mathrm{X}$ server is necessary. On the workstations, \% xhost +

On the VAX Station, we need to select "security" in the "Option" menu and define all the nodes. Then start mbone and Consumer processes.

mbone(from KEK): vtconnect nhmxw2.fnal.gov password

\section{Internet Transmission Issues}

The video and audio tools use the Internet to transmit packet multimedia data. If there are only 2 sites with no requirement to record the packet data, a simple pointto-point connection could be made. The transmitting endpoints would send data without software acknowledgement that the other end was listening. The bandwidth consumed by the isochronous audio stream is $70.1 \mathrm{kbps}$ PCM encoding. The video bandwidth is varied by the sending side and can range up to $1 \mathrm{Mbps}$. A generally acceptable quality/bandwidth compromise is $128 \mathrm{kbps}$ for video. The Internet " $\mathrm{nv"}$ video tool and "vat" audio tool programs in their basic command line form are as follows:

nv - $t$ tl $X$ machine-IP-name nv-port-number

vat $-\mathrm{t} X$ machine-IP-name/vat-port-number

where $X$ is the numerical Internet Protocol (IP) time-to-live

"machine-IP-name" is the registered DNS workstation name or IP multicast address

"port-number" is an IP/UDP datagram transport layer port number

If more than 2 sites wish to participate or there is a need to record the packets from another machine, the use of multiple streams or IP multicast is required. The Internet IP multicast infastructure known as MBONE, has been widely deployed. Its current structure uses the IP time-to-live (ttl) field to determine the scope of transmission. To reach from FNAL on ESNET to KEK would require a ttl of 127 or greater. A stream with this ttl transmitted on an MBONE without wide deployment of pruning of multicast groups would cause the $200 \mathrm{kbps}$ video/audio stream to end up in many US and overseas locations other than FNAL, KEK, or Tsukuba. Setting up a direct multicast route between sites would cause other unrelated streams to also be sent. (Recent release of multicast routing code with pruning of unwanted streams could make this appoach a possiblity in the future.)

The combination of the following requirements resulted in another network configuration choice. 
The requirements were:

- The CDF control room always sent video and audio.

- KEK and Tsukuba could also send audio and video but could leave and rejoin the conference at any time and independent of each other.

- Any part of the session could be digitally recorded at FNAL

The Multi-Session Bridge (MSB) software developed by HEPNRC was configured to bridge a combination of multicast and unicast sessions. At FNAL, the CDF control room was multicast on site only. This allowed for CDF workstations at other locations to nv_record and vat_record the sessions. The KEK and Tsukuba sites ran a program called vtconnect which informed the MSB there was an active unicast endpoint. The MSB combined the multicast and unicast streams by modifing the IP. UDP, and RTP headers to make it appear as one conference. Streams were sent over the Pacific link when KEK or Tsukuba "connected".

Future experiments should be set up to include a copy of the MSB code running at either KEK or Tsukuba. thus eliminating the need to send 2 copies of the CDF control room stream over the Pacific link when both KEK and Tsukuba are active.

\section{Recording}

Parts of the video conference were digitally recorded with v_record and vat_record. Continuous digital recording can be made via nv_record and vat_record. 10 GigaBytes of disk storage would be required for continuous recording of one weeks vat and nv packet streams.

\section{Future Plan}

We plan to have remote monitoring for the data taking Run from October, 1995 to March, 1996. The CDF control room and the adjacent meeting room are being equipped with both a PictureTel-codec and the MBONE video conference capabilities.

There have been requests to send online event pictures to education offices and other public places in Fermilab and throughout the world

Persons on data taking shift are required to record running conditions, problems, solutions and comments in the Logbook. In the 10 years of CDF history, tens of such notebooks have been produced/ One of the highly desirable improvements would be an online "Electronic Log Book".

Network links are being improved worldwide. For example, the link from Fermilab to Taiwan will be increased from the current $512 \mathrm{kbps}$ to $\mathrm{T} 1$ (1.5 Mbps) in October, 1995. This may be further increased to a T3 $(44.7 \mathrm{Mbps})$ link in the not too distant future. CDF will plan to have regular shifts from remote sites for the next Run starting in 1999. 


\section{Acknowledgments}

We thank Alvin Tollestrop. Kuni Kondo and Joel Butler for the strong push for the idea of a Remote ('ontrol Room. We thank J. Smedinghoff for making it possible for us to access Fermilab $A$ ccelerator Network and monitor the accelerators. This work is supported by the U.S. Department of Energy, the National Science Foundation, the Natural Sciences and Engineering Council of Canada. the Istituto Nazionale di Fisica Nucleare, the Ministry of Education, Science and Culture of Japan, the A.P. Sloan Foundation, the Alexander von Humboldt-Stiftung, and the National Science Council of the Republic of China 